\title{
Liquid Chromatographic Resolution of Racemic 1,4-Benzodiazepin-2-ones on Pirkle-type Chiral Stationary Phases Based on (S)-Leucine
}

\author{
Myung Ho Hyun. Yoon Jae Cho, and Jin Ah Kim \\ Department of Chemisty and Chemistry Institute for Functional Materials, Pusan National Lnirersit, Busan 609-735, Korea \\ E-mail: whhyunapusan.ack \\ Received Febriary 18, 2005
}

Key Words : Chromatographic resolution, Enantiomer separation, Chiral stationary phase, 1,4-Benzodiazepin-2-ones

It has been well known that two enantiomers of chiral drugs often show different pharmacological activity. In this instance, exact determination of the enantiomeric composition of chiral drugs is essential for the development of chiral drugs and for the better insight into the in wivo distribution and metabolism of the two enantiomers. Among various methods, liquid chromatographic separation of enantiomers on HPLC chiral stationary phases (CSPs) has been demonstrated as very attractive means for the analysis of enantiomeric composition of chiral compounds including chiral drugs. ${ }^{2}$

A series of 3-substituted 1,4-berzodiazepin-2-ones such as camazepam, lorazepam, lormetazepam and oxazepam belongs to a class of widely used anxiolytics and/or tranquilizer. ${ }^{3}$ The pharmacological activity of a series of 3substituted 1,4-berzodiazepin-2-ones has been shown to be enantiodependent. ${ }^{+}$Consequently some efforts have been devoted to the development of the liquid chromatographic separation of the two enantiomers of 3-substituted 1,4berzodiazepin-2-ones." Among others, Pirkle-type CSPs have been found to be quite useful in the resolution of racemic 3-substituted 1,4-bernzodiazepin-2-ones. Especially, CSP 1 (Figure 1) derived from (S)-N-(3,5-dinitroberzzoyl)leucine has been most successfully utilized in the resolution 3-substituted 1,4-benzodiazepin-2-ones. ${ }^{\text {5i }}$ As an improved CSP, we recently developed CSP 2 (Figure 1) by simply replacing the superfluous adsorption site, the $\mathrm{N}-\mathrm{H}$ hydrogen of the connecting tether of CSP 1, with a phenyl group. CSP 2 has shown greater enantioselectivities than CSP 1 for the enantiomers of $\pi$-acidic or $\pi$-basic derivatives of a-amino acids. $^{6 ?}$ However, CSP 2 has not been applied in the resolution of racemic 3-substituted 1,4-benzodiazepin-2ones. In this study, as an effort to extend the use of CSP 2 . we wish to elucidate that $\operatorname{CSP} 2$ is also useful in the resolution of 3-substituted 1,4-bertzodiazepin-2-ones and wish to compare the chiral recognition efficiency of $\mathrm{CSP} 2$ with that of CSP 1.

3-Substituted 1,4-benzodiazepin-2-ones ( 3 and 4 ) used in this study (Figure 1) were prepared via the reported procedure. For the purpose of comparison, 3-substituted 1,4-benzodiazepin-2-ones ( 3 and 4 ) prepared in our laboratory were resolved on CSP 1 and CSP 2 under an identical chromatographic condition and the resolution results were summarized in Table 1 . The representative chromatograms on CSP 2 are shown in Figure 2.

As shown in Table 1 and Figure 2, the resolutions of 3substituted 1,4-benzodiazepin-2-ones ( 3 and 4 ) are excellent on both CSP 1 and CSP 2 . Interestingly, the retention factors $(k)$ on CSP 2 are always smaller than those on CSP 1. Even when the content of isopropyl alcohol in mobile phase was varied within the range of $3 \%$ to $20 \%$, the retention factors ( $k$ ) on CSP 2 were still always smaller than those on CSP 1. This means that CSP 2 is better than CSP 1 in tems of reducing analytical time and saving solvent. The smaller retention factors $(k$ ) on $\mathrm{CSP} 2$ might be rationalized by considering the chiral recognition mechanism.

A chiral recognition mechanism for the resolution of 3substituted 1,4-benzodiazepin-2-ones on CSP 1 was<smiles>CCO[Si]1(CCCNC(=O)[C@H](CC(C)C)NC(=O)c2cc([N+](=O)[O-])cc([N+](=O)[O-])c2)O[C@H]2C[C@H]1C2C</smiles>

CSP 1<smiles>CC(C)C[C@H](NC(=O)c1cc([N+](=O)[O-])cc([N+](=O)[O-])c1)C(=O)N(CCC[Si](C)(O[Si](C)(C)O)C(C)C)c1ccccc1</smiles>

CSP 2<smiles>[R]C1N=C(c2ccccc2)c2ccccc2NC1=O</smiles>

3<smiles>[R]C1N=C(c2ccccc2)c2cc(Cl)ccc2NC1=O</smiles>

4

Figure 1. Structures of CSP 1, CSP 2 and 3-substituted 1,4benzodiazepin-2-ones 3 and 4 . 
Table 1. Resolution of 1,4-benzodiazepin-2-ones (3 and 4) on CSP 1 and CSP 2

\begin{tabular}{|c|c|c|c|c|c|c|c|c|c|}
\hline \multirow{2}{*}{\multicolumn{2}{|c|}{$\frac{\text { Analyte }}{\mathrm{R}}$}} & \multicolumn{4}{|c|}{ CSP 1} & \multicolumn{4}{|c|}{$\operatorname{CSP} 2$} \\
\hline & & $\mathbf{k}_{1}^{b}$ & ke & $u^{f t}$ & Cont: & $\mathrm{k}_{1}{ }^{k}$ & $k_{2}{ }^{\prime}$ & $a^{4}$ & Conf.: \\
\hline $3 \mathbf{a}$ & $\mathrm{CH}_{3}$ & 3.50 & 8.58 & 2.45 & $\mathrm{R}$ & 1.95 & 5.38 & 2.76 & $\mathrm{R}$ \\
\hline $3 \mathbf{b}$ & $\left(\mathrm{CH}_{2}\right)_{3} \mathrm{CH}_{3}$ & 2.27 & 6.95 & 3.06 & $\mathrm{R}$ & 1.56 & 4.90 & 3.14 & $\mathrm{R}$ \\
\hline $3 c$ & $\left(\mathrm{CH}_{2}\right)_{2} \mathrm{SCH}_{3}$ & 3.58 & 11.31 & 3.16 & $\mathrm{R}$ & 2.26 & 7.73 & 3.42 & $\mathrm{R}$ \\
\hline $3 d$ & $\mathrm{CH}_{2}\left(\mathrm{CH}_{3}\right)_{2}$ & 1.94 & 7.41 & 3.82 & $\mathrm{R}$ & 1.35 & 5.31 & 3.93 & $\mathrm{R}$ \\
\hline $3 e$ & $\mathrm{CH}_{2} \mathrm{CH}\left(\mathrm{CH}_{3}\right)_{2}$ & 2.16 & 7.69 & 3.56 & $\mathrm{R}$ & 1.52 & 5.46 & 3.59 & $\mathrm{R}$ \\
\hline $3 f$ & $\mathrm{CH}_{2} \mathrm{C}_{6} \mathrm{H}_{5}$ & 2.99 & 11.72 & 3.92 & $\mathrm{R}$ & 2.24 & 9.61 & 4.29 & $\mathrm{R}$ \\
\hline $4 a$ & $\mathrm{CH}_{3}$ & 2.30 & 7.06 & 3.07 & $\mathrm{R}$ & 1.35 & 3.52 & 2.61 & $\mathrm{R}$ \\
\hline $4 b$ & $\left(\mathrm{CH}_{2}\right)_{3} \mathrm{CH}_{3}$ & 1.39 & 5.32 & 3.83 & $\mathrm{R}$ & 1.05 & 3.15 & 3.00 & $\mathrm{R}$ \\
\hline $4 c$ & $\left(\mathrm{CH}_{2}\right)_{2} \mathrm{SCH}_{3}$ & 2.45 & 9.04 & 3.69 & $\mathrm{R}$ & 1.67 & 5.13 & 3.07 & $\mathrm{R}$ \\
\hline $4 d$ & $\mathrm{CH}\left(\mathrm{CH}_{3}\right)_{2}$ & 1.26 & 5.52 & 4.38 & $\mathrm{R}$ & 0.94 & 3.21 & 3.41 & $\mathrm{R}$ \\
\hline $4 e$ & $\left.\mathrm{CH}_{2} \mathrm{CH}_{2} \mathrm{CH}_{3}\right)_{2}$ & 1.46 & 5.97 & 4.09 & $\mathrm{R}$ & 1.09 & 3.55 & 3.26 & $\mathrm{R}$ \\
\hline $4 f$ & $\mathrm{CH}_{2} \mathrm{C}_{6} \mathrm{H}_{5}$ & 2.00 & 8.94 & 4.47 & $\mathrm{R}$ & 1.57 & 5.43 & 3.46 & $\mathrm{R}$ \\
\hline
\end{tabular}

Mobile phase: $10 \%$ isopropyl alcohol in hexane. Flow rate; $2.0 \mathrm{~mL} / \mathrm{min}$. Delection: $254 \mathrm{~m}$ UT. Temperature: room temperalure. "Retention factor of the lirst eluted enantiomet. TRetention factor of the second eluted enantioner. "Separation factor. "Absolute configuration of the second eluted enantiomer.

(a)

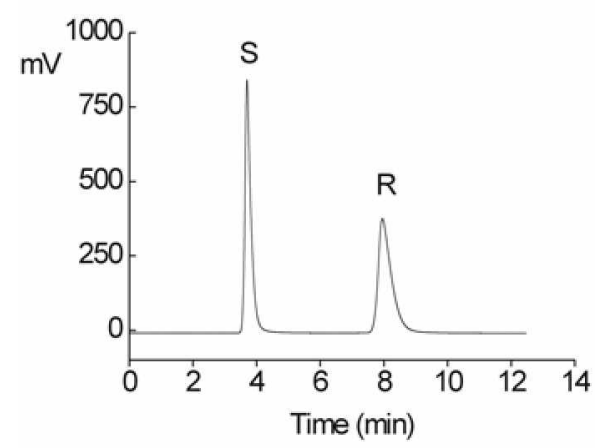

(b)

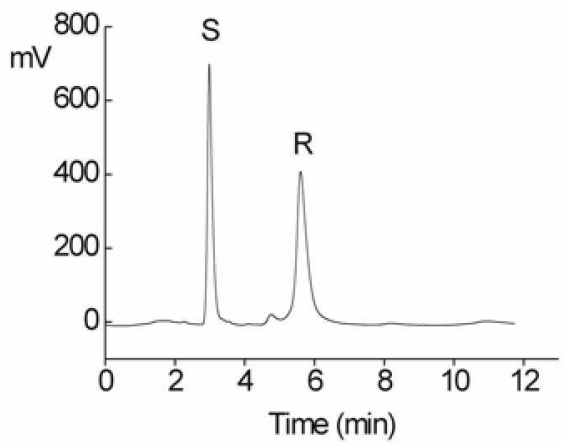

Figure 2. Representative chromatograms for the resolution of (a) 3-methyl-1,3-dihydro(2H)-5-phenyl-1,4-benzodiazepin-2-one 3a and (b) 7-chloro-3-inethyl-1,3-dihydro(2H)-5-pheny:-1,4-benzodiazepin-2-one 4 a on $\operatorname{CSP} 2$. For the chromatographic conditions, see the footnote to Table 1.

previously proposed. According to the proposed chiral recognition mechanism, the three simultaneous bonding interactions such as (a) $\pi-\pi$ donor-acceptor interaction between the 3,5-dinitrobenzoyl group of the CSP and the benzo ring of analyte, (b) hydrogen bonding interaction between the 3,5-dinitrobenzamide hydrogen of the CSP and the carbonyl oxygen of analyte and (c) hydrogen bonding of the amide hydrogen of analyte to the C-terminal carboxamide oxygen of the CSP are responsible for the chiral recognition. These interactions can occur simultaneously with the $(\mathrm{R}$ )-analytes, but not with the (S)-analytes because of the different spatial arrangement of the interaction sites and consequently, the (R)-enantioners should elute second. In the chiral recognition mechanism proposed, the $\mathrm{N}-\mathrm{H}$ hydrogen of the tethering anide group of CSP 1 does not play any significant role. Instead, the N-H hydrogen of the tethering annide group of CSP 1 is expected to increase the retention of analytes because of the non-enantioselective hydrogen bonding interaction with analytes. In this instance, the retention factors $(k)$ are expected to be smaller on CSP 2, which does not contain a superfluous hydrogen bonding $\mathrm{N}-\mathrm{H}$ site, compared with those on CSP 1.

The retention factors $(k)$ for the resolution of 3-substituted 1,4-bemzodiazepin-2-ones $\mathbf{3}$ are also observed to be always greater than those for the resolution of 3-substituted 1,4benzodiazepin-2-ones 4 both on CSP 1 and CSP 2 as shown in Table 1. According to the chiral recognition mechanism, $\pi-\pi$ donor-acceptor interaction between the $\pi$-acidic 3,5dinitrobenzoyl group of the CSP and the $\pi$-basic benzo ring of analyte is very important for the chiral recognition. The strength of $\pi-\pi$ donor-acceptor interaction between the 3,5dinitrobenzoyl group of the CSP and the benzo ring of analyte is expected to be dependent on the strength of the $\pi$-basicity of the benzo ring of analytes. In this instance, 3substituted 1,4-benzodiazepin-2-ones 3 , which contain relatively more $\pi$-basic non-substituted benzo ring, should be retained longer than 3-substituted 1,4-benzodiazepin-2ones 4 , which contain relatively less $\pi$-basic chlorinesubstituted belzo ring.

Even though the separation factors $(\alpha)$ are large enough for the chiral analysis on both CSP 1 and CSP 2, the trends of the separation factors $(\alpha)$ on the two CSPs with the change of the substituent on the benzo ring of analytes from hydrogen to chlorine are quite interesting. CSP 1 shows 
greater separation factors $(\alpha)$ for the resolution of 3 substituted 1,4-benzodiazepin-2-ones 4 than for the resolution of conresponding 3-substituted 1,4-berzodiazepin-2-ones 3 . In contrast, CSP 2 shows greater separation factors $(\alpha)$ for the resolution of 3-substituted 1,4-bernzodiazepin-2-ones 3 than for the resolution of corresponding 3 substituted 1,4-benzodiazepin-2-ones 4. In consequence, CSP 2 shows greater separation factors ( $\alpha$ ) for the resolution of 3-substituted 1,4-benzodiazepin-2-ones 3 than CSP 1 does while CSP 1 shows greater separation factors $(\alpha)$ for the resolution of 3-substituted 1,4-benzodiazepin-2-ones 4 than CSP 2 does. Based on the chiral recognition mechanism proposed, 3-substituted 1,4-benzodiazepin-2-ones 3 was expected to be resolved better than corresponding 3substituted 1,4-benzodiazepin-2-ones 4 on both CSP 1 and CSP 2 because the $\pi=\pi$ interaction between the 3,5 dinitrobenzoyl group of the CSP and the benzo ring of analyte should be greater with the non-substituted berzo ring than with the chlorine-substituted berzo ring. Namely, the effect of the substituent on the benzo ring of anlylytes on enantioselectivity should be equal on the two CSPs based on the chiral recognition mechanism. However, this expectation was turned out to be true with only CSP 2, not with CSP 1. By replacing the $\mathrm{N}-\mathrm{H}$ hydrogen of the connecting tether of CSP 1 with a phenyl group in CSP 2, the conformation of CSP 2 should be altered from that of CSP 1. The subtle conformational difference between CSP 1 and CSP 2 might be responsible for the unexpected results of the separation factors (a) on the two CSPs. However, the exact reason for these unexpected results is not clear yet.

In summary, CSP 2, which was developed in our laboratory as an improved version of CSP 1, was demonstrated to be more attractive than CSP 1 in the resolution of 3-substituted 1,4-benzodiazepin-2-ones in terms of reducing analytical time and saving eluting solvent. In addition, CSP 2 was concluded to be equally effective as CSP 1 in the resolution of 3-substituted 1,4-benzodiazepin-2-ones in terms of enantioselectivity. However, the trends of the separation factors $(\alpha)$ on the two CSPs were not consistent with each other with the change of the substituent on the benzo ring of analytes. Even though the conformational difference between CSP 1 and CSP 2 is expected to be responsible for the non-consistent trends of the separation factors $(\alpha)$ on the two CSPs with the change of the substituent on the benzo ring of analytes, in order to elucidate the exact reason for these unexpected results, the study for the molecular interaction between the CSP and the analyte is underway in our laboratory.

\section{Experimental Section}

Chromatography was performed with an HPLC system consisting of a Waters model 510 pump, a Rheodyne model $7125 \mathrm{i}$ injector with a $20 \mu \mathrm{L}$ sample loop, a YoungLin M720 absorbance detector with a $254 \mathrm{fm}$ UV filter and a YoungLin Autochro Data Module (Software: YoungLin AutochroWIN 2.0 plus). The chiral column (Pirkle Covalent Leucine,
$250 \times 4.6 \mathrm{~mm}$ ID) packed with CSP 1 was commercially available from Regis Chemical Company (Morton Glove, IL, USA). The chiral column $(250 \times 4.6 \mathrm{~mm}$ ID) packed with CSP 2 was available from the previous study. All chromatographic experiments were carried out at a flow-rate of $2.0 \mathrm{~mL} / \mathrm{min}$ at $20^{\circ} \mathrm{C}$. The void volume was detemined by the injection of 1,3,5-tri-tert-butylbenzene. Racemic and optically active 3-substituted 1,4-benzodiazepin-2-ones (3 and 4) used in this study were prepared by coupling $o$ aminobenzophenones with racemic or optically active $N$-tBoc- $\alpha$-amino acids followed by deprotection and cyclization in the presence of trifluoroacetic acid via the known procedure. ${ }^{8}$ The structures of 3-substituted 1,4-benzodiazepin-2-ones ( 3 and 4 ) prepared were well in accordance

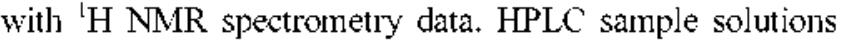
were prepared by dissolving each 3-substituted 1,4-benzodiazepin-2-one in methylene chloride $(1.0 \mathrm{mg} / \mathrm{mL})$. Injection volume of each sample was usually $2 \mu \mathrm{L}$. The elution orders shown in Table 1 were determined by comparing the chromatograms for the racemic and configurationally known samples prepared from racemic and optically active N-rBoc- $\alpha$-amino acids, respectively.

Acknowledgements. This work has been supported by a grant from KISTEP (NRL Program: M1-0318-00-0005).

\section{References}

1. Crossley, R. Chirality and the Biological Activity of Drtes; CRC Press: Boca Raton, 1995.

2. (a) Beesley, T. E.: Scott, R. P. W. Chiral Chomatograpln; John Wiely \& Sons: New York, 1998. (b) Ahuja, S. Chimal Separations by. Chronatograph: American Chemical Society, oxford University Press: Oxford, 2000. (c) Aboul-Enein, H. Y.: Ali, I. Chiral Separations br Ligtid Chrontatograph and Related Technologies; Marcel Dekiker: New York. 2003. (d) Kim. I. W.; Ryu, J. K.; Ahn, S. D.; Park, J. H.; Lee, K.-P.; Ryoo, J. J.; Hyun, M. H.; Okamoto, Y.; Yamainoto, C.; Carr, P. W. Bull. Koreart Chem. Soc. 2003, 24, 239. (e) Hyun, M. H.; Min, H. J.; Cho, Y. J. Bull. Korean Chem. Soc. 2003, 24, 91 l. (1) Hyun, M. H.: Kim, Y. H.: Cho, Y. J. Bull. Korean Chem. Soc. 2004, 25, 1707 . (g) Hyun, M. H.: Cho, Y. J.: Choi, H. J.; Lee, K. W. Bull. Korean Chem. Soc. $2004,25,1977$

3. (a) Mennini, T.; Caccia, S.; Garattini, S. Prog. Drig. Res. 1987 , 31, 315. (b) Kleemann, A.; Engel, J. Phomacentical Substances, 3rd ed; Thieme: Stuttgart, 1999.

4. (a) Sunjic, V.; Dejanovic, R.; Palkovic, A.; Klasinc, L.; Kajfez, F. Tetrahedror Lett. 1076, 4493. (b) Savadori, P.; Bertucci, C.; Donnenici, E.: Giannaccini, G. J. Pham Bioned. Anal. 1989, 7 , 1735 .

5. (a) Pirkle, W. H.: Tsipouras, A.J. Chomatogr: 1984, 291, 291. (b) Bertucei, C.; Uccello-Barretla, G: Savadori, P. J. Chronatogr: 1990, 506, 617. (c) Katli, A.; Erlandsson, P.: Dappen, R. J. Chonktogr. 1992, 590, 127. (d) Oliveros, L.; Minguillon, C.; Billaud, C.J. Pharam. Bioned. Anol. 1992, 10,925. (e) Lu, X.-L.; Yang, S. K. $J$. Chromatogr. $A$ 1994, 606, 249.

6. Hyun, M. H.; Lee, J. B.; Kim, Y. D. J. High Resol. Chromatogn: $1998,2 f, 69$.

7. (a) Hyun, M. H.; Lee, S. J.; Ryoo, J.-J. Buhl. Korean Chem. Soc. $1998,19,1105$, (b) Hyun, M. H.; Kang, M. H.: Han. S. C. J. Chromatogr: A 2000, 868,39 . (c) Hyun, M. H.: Cho, Y. J.: Baik, I.-K. Bull. Korew Chem. Soc. 2002, $23,1291$.

8. Sunjic, V.: Kajfez, F.; Stromar, I.: Blazevic, N.: Kolbah, D. $J$. Heteroc7cl. Chem. 1973, 10,591. 